\title{
RETRIEVAL OF AEROSOL OPTICAL PROPERTIES BASED ON HIGH SPECTRAL RESOLUTION LIDAR
}

\author{
Da Xiao ${ }^{1}$, Tianfen Zhong ${ }^{1}$, Xue Shen ${ }^{1}$, Nanchao Wang ${ }^{1}$, Yuhang Rong ${ }^{1}$, Chong Liu ${ }^{1}$, Yupeng \\ Zhang $^{1}$, Dong Liu ${ }^{*}$ \\ ${ }^{1}$ State Key Laboratory of Modern Optical Instrumentation, College of Optical Science and Engineering, \\ Zhejiang University, Hangzhou 310027, China \\ Email:*liudongopt@zju.edu.cn
}

\begin{abstract}
The detection of clouds and aerosols is important for climate research. Lidar has been widely used in atmospheric remote sensing research because of its high spatial and temporal resolution and ability to detect profiles. High spectral resolution lidar (HSRL) accurately calculates the optical properties of aerosols and clouds without relying on any assumptions. Based on the $532 \mathrm{~nm}$ iodine HSRL system, the lidar ratio of the urban aerosol in Hangzhou is 40-50sr, and the average lidar ratio of the cirrus is $24.79 \mathrm{sr}$, demonstrating that the HSRL system and retrieval algorithms accurately obtain the optical properties of clouds and aerosols.
\end{abstract}

\section{INTRODUCTION}

Aerosols and clouds play an important role in global and local climate and becoming two of the largest uncertainty sources in climate sensitivity research [1, 2]. Aerosols directly affect the solar and terrestrial radiation in the atmosphere by scattering and absorbing light, influencing the Earth's radiation budget, while clouds play a major role in determining The Earth's climate [3]. The acquisition of cloud and aerosol optical parameters and vertical profile information is of great significance for atmospheric research.

Lidar, regarded as the only means to obtain the vertical profile distribution characteristics of clouds and aerosols, has been widely used. The traditional lidar cannot directly calculate the extinction coefficient. The extinction coefficient can only be calculated with the assumption of the ratio of the extinction coefficient to the backscatter coefficient [4]. However, the aerosol lidar ratio is a highly variable quantity, so that large errors in the retrieval must be expected if the lidar ratio is not known exactly [5]. Raman lidar and high spectral resolution lidar (HSRL) can accurately invert the extinction coefficient without any assumptions. Raman lidars are limited by low signal-to-noise ratios and require long time averaging, which is not suitable for daytime measurements [6]. The HSRL system cleverly uses the spectral difference of the lidar echo signals to separate the aerosol return from the molecular return through a spectral discriminator with high spectral resolution, so the extinction coefficient and lidar ratio can be accurately measured.

This paper briefly introduces the principle of retrieval and describes the process of data retrieval to obtain the optical properties of atmospheric aerosols in detail.

\section{METHODOLOGY}

\subsection{The principle of retrieval}

A general 532nm iodine HSRL system block diagram with abstract structure is shown in Fig.1. A bundle of parallel light received by the receiving system is split by the PBS, and the reflected beam is received by a PMT as a mixed vertical channel. The PBS transmitted light consists of parallel components, which are split by a splitting prism (BS), and the reflected light is received by the detector as a mixed parallel channel. The transmitted light of the BS passes through an iodine pool and is received by the detector as the molecular channel.

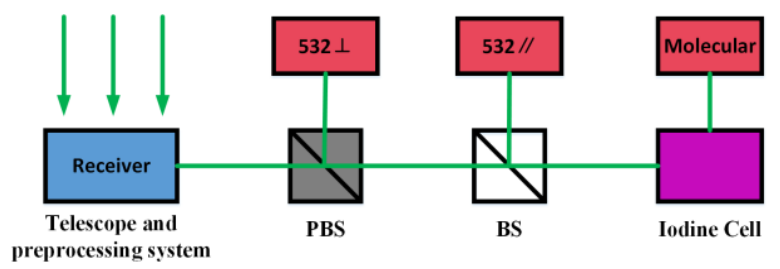

Fig.1 A general 532nm iodine HSRL system block diagram with abstract structure.

The echo signals received by the three detectors can be represented by Equation 1.1. 


$$
\begin{aligned}
& B_{c}^{\perp}=\left(\beta_{a}^{\perp}+\beta_{m}^{\perp}\right) e^{(-2 \tau)} \\
& B_{c}^{\prime \prime}=\left(\beta_{a}^{\prime \prime}+\beta_{m}^{\prime \prime}\right) e^{(-2 \tau)} \\
& B_{m}^{\prime \prime}=\left(\beta_{a}^{\prime \prime} T_{a}+\beta_{m}^{\prime \prime} T_{m}\right) e^{(-2 \tau)}
\end{aligned}
$$

where $B_{c}^{\perp}, B_{c}^{\prime \prime}$ and $B_{m}^{\prime \prime}$ are the attenuation scatter signals obtained by the mixed vertical channels, mixing the parallel channels and the molecular channels, respectively. $\beta_{a}$ and $\beta_{m}$ are the backscattering coefficients of aerosol and atmospheric molecules, respectively. The superscripts " $\perp "$ and $" / / "$ indicate their vertical polarization and parallel polarization components, respectively. $\tau$ is the atmospheric optical thickness, $T_{m}$ and $T_{a}$ are the transmittances of the spectral discriminator for the molecular scattering signal and the aerosol scattering signal, respectively.

Combining the three signals, we can get the atmospheric backscatter coefficient

$$
\beta=\beta_{m} \frac{(1+\delta)\left(T_{m}-T_{a}\right) K}{\left(1+\delta_{m}\right)\left(1-T_{a} K\right)}
$$

Where $\delta_{m}$ is the depolarization ratio of atmospheric molecules, which can be accurately obtained with atmospheric model. $\delta$ is the total depolarization ratio of atmospheric molecules and aerosols, which can be obtained by the ratio of the mixed vertical channel to the mixed parallel channel, as in Equation 1. 3.

$$
\delta=\frac{B_{c}^{\perp}}{B_{c}^{\prime \prime}}
$$

The definition of $\mathrm{K}$ is similar to the depolarization ratio, which can be obtained by the ratio of mixed parallel channels to molecular channels, as in Equation 1.4.

$$
K=\frac{B_{c}^{\prime \prime}}{B_{m}^{\prime \prime}}
$$

Meanwhile, we can get the expression of atmospheric optical thickness in Equation 1.5.

$$
\tau=-\frac{1}{2} \ln \left(\frac{\left(1-T_{a} K\right)\left(1+\delta_{m}\right) B_{m}^{\prime \prime}}{\left(T_{m}-T_{a}\right) \beta_{m}}\right)
$$

After obtaining the basic quantities $\beta$ and $\tau$, we can perform the retrieval of the aerosol extinction coefficient $\alpha_{a}$, as in Equation 1.6.

$$
\alpha_{a}=\frac{\partial \tau}{\partial r}-\alpha_{m}
$$

Where $\alpha_{m}$ is the extinction coefficient of atmospheric molecules.

\subsection{Retrieval algorithm and process}

A HSRL system based on $532 \mathrm{~nm}$ iodine cell has been set up by Zhejiang University. Based on this system, we developed a retrieval algorithm for calculating the optical properties of atmospheric aerosols, as shown in Fig.2.
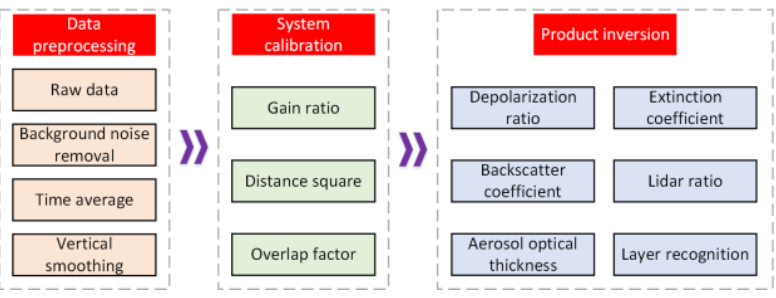

Fig.2 HSRL retrieval algorithm block diagram

The retrieval algorithm is divided into three modules, data pre-processing, system calibration, and product retrieval. The purpose of the data preprocessing module is to remove background noise and improve signal to noise ratio. The original lidar echo signal can be represented by Equation 1.7 .

$$
S(z, \lambda)=S_{a}(z, \lambda)+S_{m}(z, \lambda)+S_{a t m}(\lambda)+S_{e l}
$$

Where $S_{a}(z, \lambda)$ and $S_{m}(z, \lambda)$ are the signal contributions backscattered by aerosol particles (a) and molecules $(\mathrm{m})$ at altitude $\mathrm{z}$ and at wavelength $\lambda, S_{a t m}(\lambda)$ is the optical signal background from the atmosphere, which is independent of range, $S_{e l}$ represents circuit background noise, depending on the electronic effects of signal detection and data acquisition. The constant background components $S_{a t m}(\lambda)$ and $S_{e l}$ can be determined by the long range of lidar signals, far enough to make the expected contribution of atmospheric backscatter negligible, or to utilize the pre-trigger range before the laser pulse [7].

Time integration and vertical smoothing are used to reduce noise and improve signal to noise ratio. The time average is limited to around 1000 profiles to ensure adequate high time resolution. For vertical smoothing, there are common 
methods such as wavelet denoising, EMD noise reduction, S-G filtering and Butterworth filtering.

System calibration is used to calibrate the effects of system parameters on the retrieval results, which includes the gain of each channel, overlap factor and the distance squared correction. The gain ratio correction for each channel eliminates the effects of different detectors and acquisition amplifier circuits on signal gain. Distance correction primarily considers the trigger delay effect due to the delay in the timing of the laser output pulse and the timing at which the digital acquisition system begins recording the laser profile. The correction of the overlap factor helps to correctly invert the near-field aerosol. In the HSRL system, accurate measurement of the overlap factor is a problem that is significant for the retrieval of optical thickness and extinction coefficient. For the HSRL system developed by Zhejiang University, to correct the overlap factor effectively, we specially add an auxiliary channel with a telescope in small size, which is shown in Fig.4.

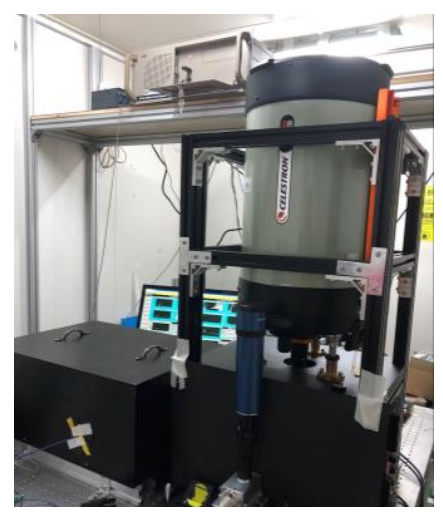

Fig 4 A receive module schematic diagram of HSRL

As mentioned before, HSRL has the advantage of inverting aerosol optical parameters without any

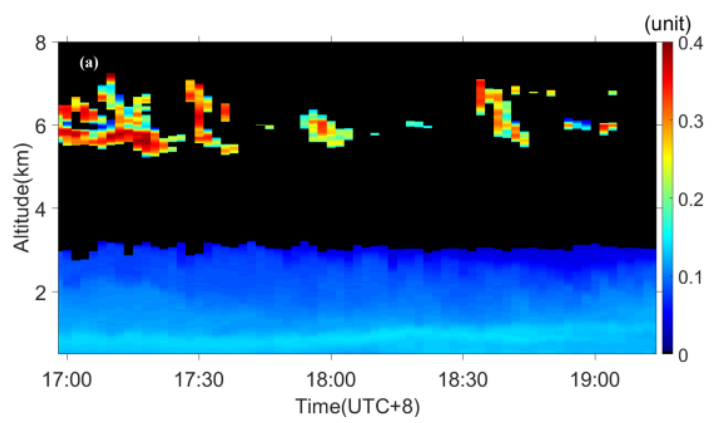

assumptions. A polarized HSRL system can not only calculate the optical characteristic parameters of the particles, such as backscattering coefficient and extinction coefficient, but also invert the depolarization ratio and extinction-to-backscatter ratio that reflect the microscopic physical properties of the particles. Extinction coefficient, obtained by the derivative of the optical thickness, is the main cause of the fluctuation of lidar ratio. A Savitzky-Golay filter of first order is used for the numerical derivative of the AOT profile [5]. This is equivalent to a linear fit of the specified number of points in the moving window, with the slope of the fitted line for each point as the derivative value.

With the obtained optical properties, we can get more accurate other atmospheric information, such as boundary layer and atmospheric layer information.

\section{RESULTS}

With long-term observation HSRL data, we verify the feasibility of the retrieval algorithm. Figure 3 shows the data retrieval result during the measurement on March 12, 2019 at the Yuquan Campus of Zhejiang University. Figure 3(a)-(d) are the depolarization ratio, the backscattering coefficient, the extinction coefficient, and the extinction-to-backscatter ratio, respectively. There is a strong aerosol load below $3 \mathrm{~km}$, and there is a cloud at an altitude of about $6 \mathrm{~km}$. The average backscatter coefficient and depolarization ratio is $0.004 \mathrm{~km}^{-1} \mathrm{sr}^{-1}$ and 0.1 in aerosol layer, respectively. The average value of the extinction coefficient is $0.18 \mathrm{~km}^{-1}$, and the lidar ratio of the urban aerosol in Hangzhou is $40-50 \mathrm{sr}$. For the cloud layer, the values are $0.37,0.04 \mathrm{~km}^{-1} \mathrm{sr}^{-1}, 1.02 \mathrm{~km}^{-1}, 24.80 \mathrm{sr}$, respectively..





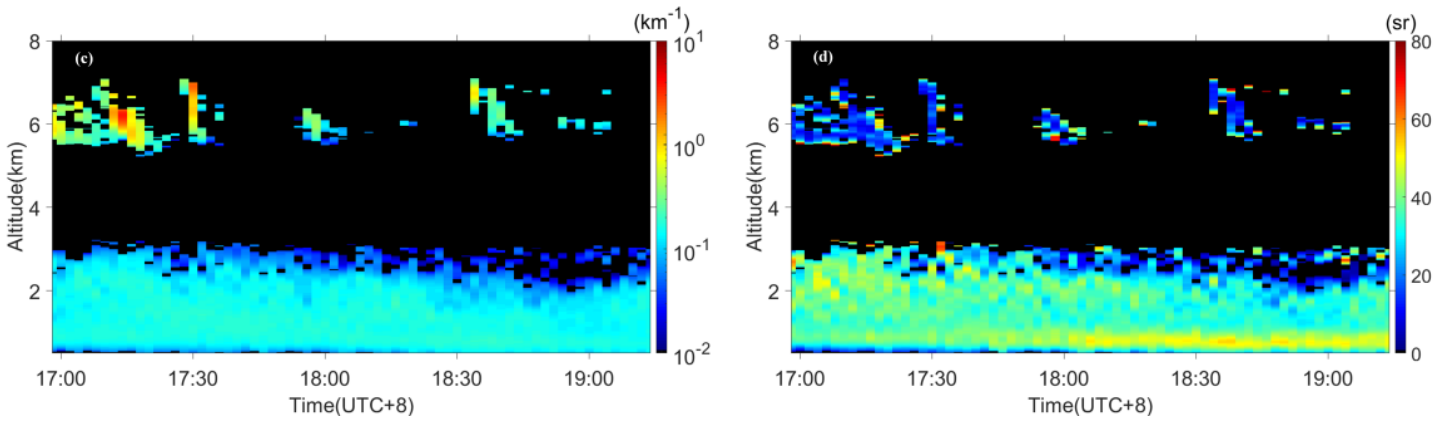

Fig 3 The data retrieval result during the measurement on March 12, 2019 at the Yuquan Campus of Zhejiang University. These four pictures are: (a) the depolarization ratio; (b) the backscatter coefficients; (c) the extinction coefficients; (d) the extinction-to-backscatter ratio.

The results are in good agreement with the data range of urban aerosols and cirrus clouds, which demonstrates that the HSRL system and retrieval algorithms accurately obtain the optical properties of clouds and aerosols.

\section{ACKNOWLEDGEMENTS}

This work was supported by the National Key Research and Development Program of China (2016YFC0200700, 2016YFC1400905), National Natural Science Foundation of China (41775023, 41305023, 61475141), Natural Science Foundation of Zhejiang Province (LR19D050001).

\section{REFERENCES}

[1] Liou, K. N. . "An Introduction to Atmospheric Radiation." Physics Today 34.7(1981):66-67.

[2] Mao, Feiyue , W. Gong, and Z. Zhu . "Simple multiscale algorithm for layer detection with lidar." Applied Optics 50.36(2011):6591-8.

[3] Hair, J. W. , et al. "Airborne high spectral resolution lidar for profiling aerosol optical properties. " Applied Optics 47.36(2008):6734-52.

[4] Klett, James D. . "Stable analytical retrieval solution for processing lidar returns." Applied Optics 20.2(1981):211-20.

[5] Esselborn, Michael, et al. "Airborne high spectral resolution lidar for measuring aerosol extinction and backscatter coefficients." Applied Optics 47.3(2008):346-358.

[6] Liu, Dong, et al. "Retrieval and analysis of a polarized high-spectral-resolution lidar for profiling aerosol optical properties." Optics Express 21.11(2013):13084.

[7] D'Amico, Giuseppe, et al. "EARLINET Single Calculus Chain - technical - Part 1: Pre-processing of raw lidar data." Atmospheric Measurement Techniques,9,2(2016-02-12) 8.10(2016):10387-10428. 